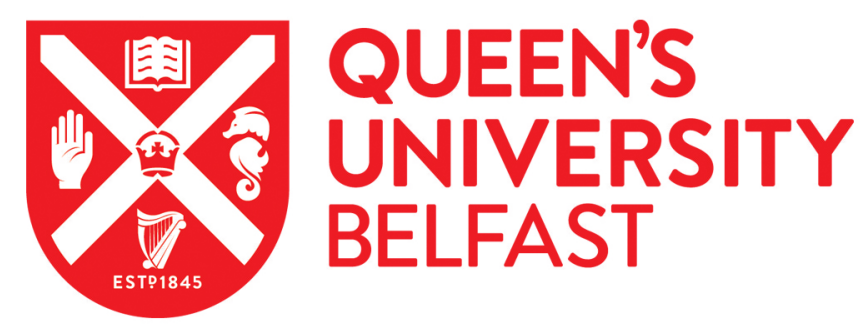

\title{
Tracking dynamics of retrodirective vehicle RADAR with track hold ability
}

Buchanan, N., Ding, Y., \& Fusco, V. (2017). Tracking dynamics of retrodirective vehicle RADAR with track hold ability. Paper presented at International Conference on Radar Systems, Belfast, United Kingdom.

\section{Document Version:}

Early version, also known as pre-print

Queen's University Belfast - Research Portal:

Link to publication record in Queen's University Belfast Research Portal

\section{Publisher rights}

@2017 The Institution of Engineering and Technology. This work is made available online in accordance with the publisher's policies. Please refer to any applicable terms of use of the publisher.

\section{General rights}

Copyright for the publications made accessible via the Queen's University Belfast Research Portal is retained by the author(s) and / or other copyright owners and it is a condition of accessing these publications that users recognise and abide by the legal requirements associated with these rights.

Take down policy

The Research Portal is Queen's institutional repository that provides access to Queen's research output. Every effort has been made to ensure that content in the Research Portal does not infringe any person's rights, or applicable UK laws. If you discover content in the Research Portal that you believe breaches copyright or violates any law, please contact openaccess@qub.ac.uk. 


\title{
Tracking dynamics of retrodirective vehicle RADAR with track hold ability
}

\author{
N.B. Buchanan, Y. Ding, V. Fusco \\ The Institute of Electronics, Communications and Information Technology (ECIT), Queen's University Belfast, Northern \\ Ireland Science Park, Queen's Road, Queen's Island, Belfast, United Kingdom, BT3 9DT, Tel +44 2890 971721, Fax +44 28 \\ 90971702 \\ n.buchanan@ecit.qub.ac.uk
}

Keywords: Radar, Radar tracking, Antenna arrays, Adaptive Arrays.

\begin{abstract}
This paper considers the tracking dynamics of a retrodirective RADAR when applied to a vehicular scenario. The advantages of such a RADAR system are discussed in comparison to a conventional RADAR. More specifically, a "track hold" function is presented for a retrodirective RADAR, which is shown, for typical vehicle turning rates, to be able to maintain the retransmitted beam towards the target during dropouts in the reflected signal. These dropouts could be caused by effects such as variations of target radar cross section with angle of incidence. The "track hold" feature prevents the need for a reacquisition of the target after the dropout and also provides the possibility of using the RADAR to reliably transmit data to the target. The ability to provide a reliable retransmitted beam, even in the absence of a target reflected signal, is a significant improvement compared to traditional mixer based retrodirective antennas, which are unable to maintain the retransmitted signal in the absence of a received signal.
\end{abstract}

\section{Introduction}

Retrodirective RADAR has the advantage of fast acquisition and tracking of a target using relatively simple circuits. There is no requirement for mechanically, or electronically scanned directional antennas as would be the case in a conventional RADAR. In conventional RADAR, a directional antenna is scanned, by mechanical or electronic means, and a pulsed signal is transmitted, with the return (echo) signal providing the range information. Direction information is obtained from the position of the scanning antenna, or computed angle of arrival, at the time the return pulse from a target was received. In a retrodirective RADAR there is no requirement to mechanically, or electronically, scan the antenna, since the target is acquired simply by a phase conjugation process that ensures that the maximum retransmitted beam is always focused onto the target. The retransmitted phase values can then be used to calculate the position of the target. During the absence of a target the retrodirective RADAR transmits a broad illumination, but can quickly reacquire, and refocus the beam when a target appears in the field of view.
One major advantage of the retrodirective RADAR is the speed and simplicity of tracking, since, once the RADAR has acquired the target, it stays locked to it, rather than continuously scanning as is the case of a conventional RADAR, where the tracking speed would be limited by the scanning speed. It has been shown that a retrodirective RADAR can track fast moving targets (of up to $780 \mathrm{~ms}$ ) [1]. The retrodirective RADAR has also been practically shown to successfully detect the position of near field targets [2].

In this paper we look at the aspects behind applying a retrodirective RADAR to a vehicular scenario. In this application mass produced retrodirective RADARs can benefit from simple circuitry, which is typically small enough to be incorporated entirely within the space occupied by the antenna array. One of the main challenges that will be addressed in this paper is the ability of the retrodirective RADAR to continuously stay locked to a target (using a track hold function), even if dropouts are received from the reflected signal. Such dropouts could occur due to variations in the targets' radar cross section (RCS) with azimuth angle. The advantage of having the retrodirective RADAR continuously locked to the target, even during reflected signal dropouts, provides for consistent tracking with no reacquisitions needed. The retrodirective antenna has also been previously shown to be able to operate with modulated signals [3], that have little, or no effect on the retrodirective operation, meaning that the RADAR could also be used to reliably transmit/receive data to the target without any interruptions to the retransmitted beam. The applications of this retrodirective RADAR are particular pertinent due to the potential widespread use of autonomous vehicles.

\section{Retrodirective RADAR with "Track Hold" function}

In this section the tracking dynamics of a retrodirective RADAR applied in a vehicular RADAR scenario are studied. In particular, the effects on the retrodirective RADAR tracking ability if a dropout is received on the reflected signal from the target. A likely cause of such a dropout is the variation of RCS of the target as the angle of incidence is varied. Considerable measured data showing this phenomenon is available (eg [4]). This measured data shows 
the reflected signal from a vehicle can vary by up to $40 \mathrm{~dB}$ over the measured azimuth range. Typically the highest reflected signal is experienced when most of the flat panels of the vehicle are at normal incidence to the RADAR and will reduce significantly as the RADAR is moved away from normal incidence.

Employing a traditional (mixer based) retrodirective RADAR [5] would mean that the tracking ability is almost instantly lost during the dropout of a reflected signal since the mixer based architecture cannot maintain the transmit signal direction in the absence of received signal. More recently QUB has provided improvements to the traditional mixer based retrodirective antennas by the addition of a tracking PLL [6]. PLL based retrodirective antennas can offer some degree of "flywheel" effect when a dropout is received, which provides some ability to hold the tracking position, until the target signal reappears. Hold times of up to $0.27 \mu$ s have been practically shown in pulsed retrodirective RADARs [7]. Longer hold times could be possible by using slower time constant loop filters, but would still be likely to be restricted to periods of several ms. Newer development to this PLL architecture, shown in Fig 1, involves adding a microcontroller to the tracking loop, which provides a "track hold" function of several seconds. Other advantages of this approach, include straightforward obtaining of the target position information, as this is easily calculated from the array phase information from the microcontroller.

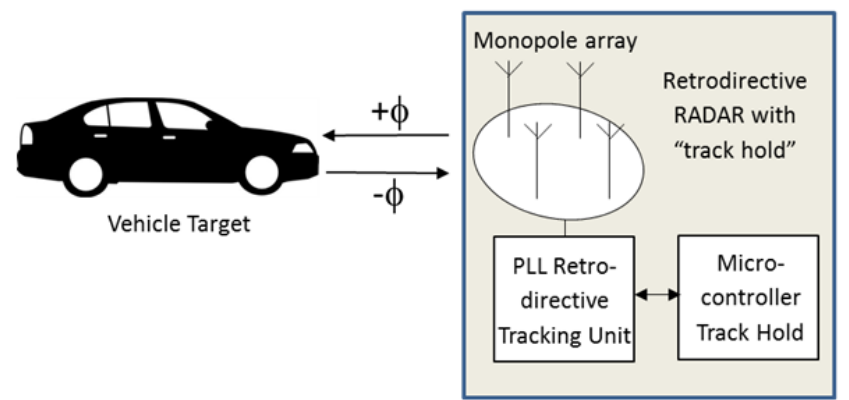

(a) Retrodirective RADAR with track hold - block diagram

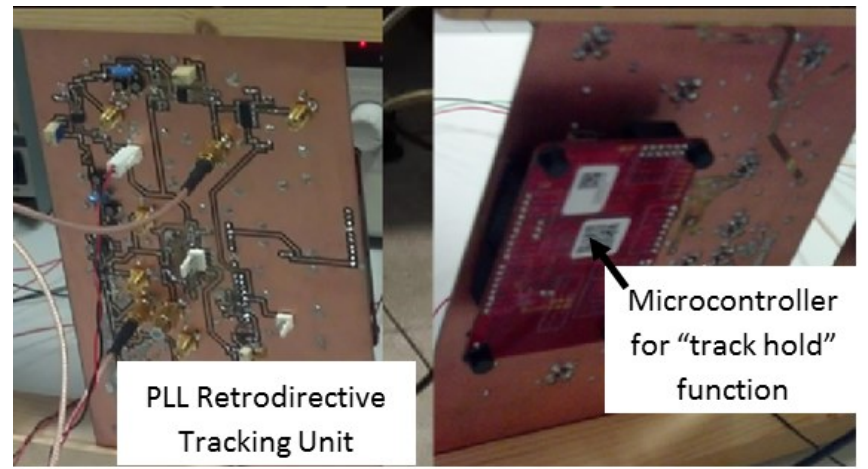

(b) Practical realisation of a single tracking unit with microcontroller

Fig. 1: Retrodirective RADAR with track hold

\section{Tracking dynamics for a typical mobile scenario}

Calculations are now shown, for a retrodirective RADAR mounted on a moving vehicle, which experiences a dropout from the target reflection, for a certain time period. The aim here is to determine, when the target reappears, if it is still within the half power beamwidth of the RADAR's antenna array. This would mean that if the RADAR was able to "track hold" during a target reflection dropout, then a maximum beam (within a certain tolerance) would still be returned to the target during the period of the dropout in the reflected signal. This would have the advantage that a reacquisition would not be required when the target reappears. In addition, if the RADAR signal was also used to carry data, there would be no loss of transmitted data, during the period of loss of target reflection.

By considering the beamwidth of different antennas, at different frequencies, we estimate, if the reflected signal disappears, the time period for the retransmitted beam to reach the $3 \mathrm{~dB}$ points. Three different antenna configurations are considered, at $400 \mathrm{MHz}, 2.4 \mathrm{GHz}$ and $5 \mathrm{GHz}$. Circular monopole arrays are used for the antenna configuration, as these offer $360^{\circ}$ azimuthal coverage. Figure 2 (a-c) show the antenna array configurations. $3 \mathrm{~dB}$ beam widths are predicted to be $60^{\circ}, 10^{\circ}$ and $5^{\circ}$ at $400 \mathrm{MHz}, 2.4 \mathrm{GHz}$ and $5 \mathrm{GHz}$ respectively.

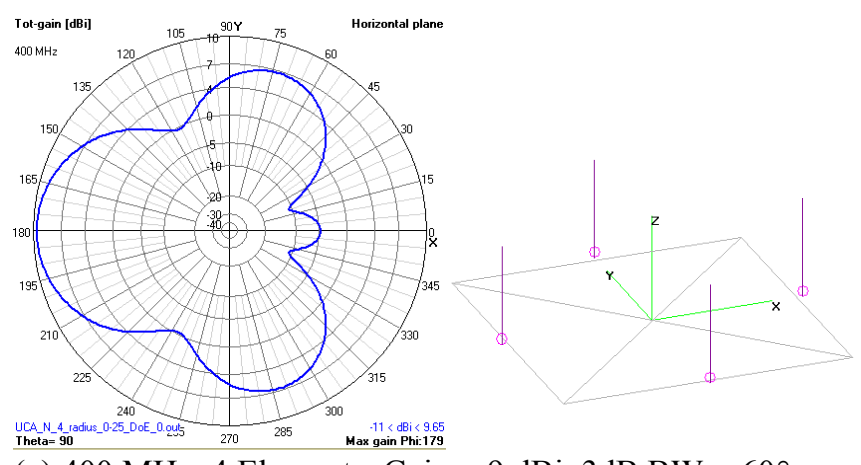

(a) $400 \mathrm{MHz}, 4$ Elements, Gain $=9 \mathrm{dBi}, 3 \mathrm{~dB} \mathrm{BW}=60^{\circ}$

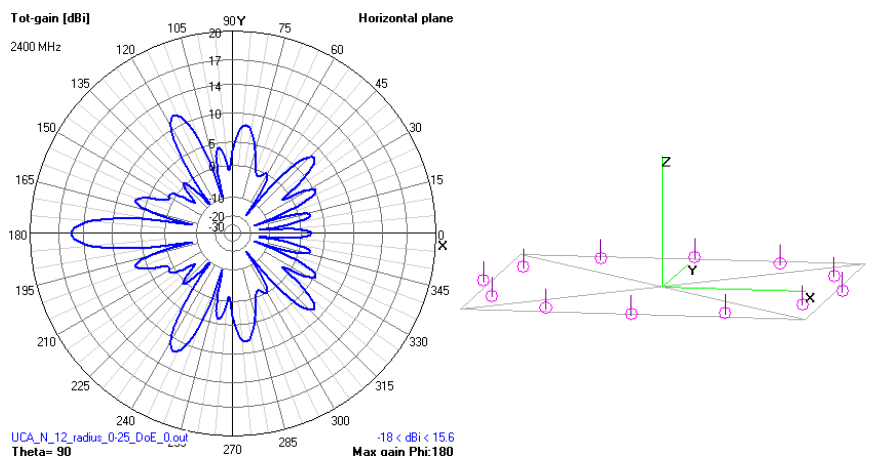

(b) $2.4 \mathrm{GHz}, 12$ Elements, Gain $=15.6 \mathrm{dBi}, 3 \mathrm{~dB} \mathrm{BW}=10^{\circ}$ 


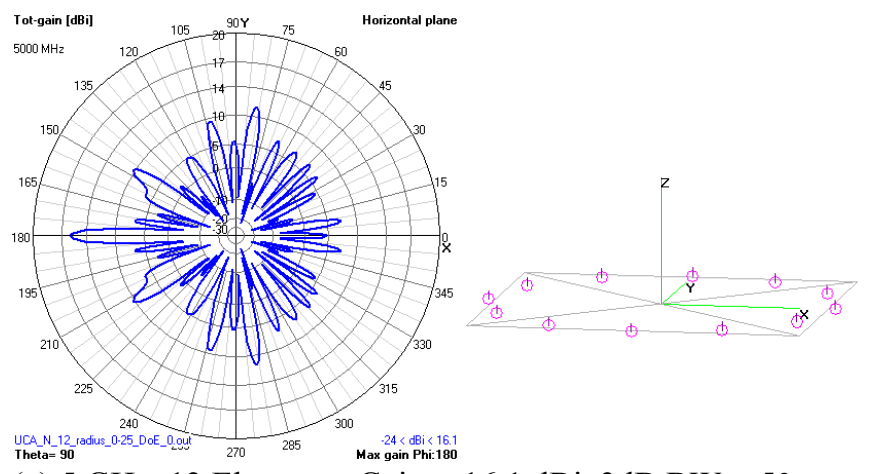

(c) $5 \mathrm{GHz}, 12$ Elements, Gain $=16.1 \mathrm{dBi}, 3 \mathrm{~dB} \mathrm{BW}=5^{\circ}$

Fig. 2: Retrodirective RADAR array configurations.

The calculations in this section are based on a retrodirective RADAR mounted on a vehicle experiencing a certain turning rate. Turning rates of up to $60 \%$ are used for the calculations, which are taken from typical specifications for mobile steerable antennas [8]. Using the predicted beamwidths from Figure 2, it is possible to calculate the maximum time $(T)$ that a signal dropout from the target reflection can be experienced, before the signal moves beyond the $3 \mathrm{~dB}$ beam width of the antenna array. This can be calculated from:

$$
T=\frac{3 d B \text { beamwidth }}{2(\text { Turning Rate })}
$$

Therefore if the target reflected signal re appears in less than this time period then it is assumed that the retrodirective RADAR has been able to maintain the retransmitted beam within $3 \mathrm{~dB}$, towards the target, during the drop out period. If this is the case, then a "track hold" function that can hold the target direction during a dropout will suffice, to maintain the retransmit signal to the target.

These calculations from equation (1) are plotted on the graph of Fig 3. From Fig 3 it can be seen that the target dropout time $(T)$ varies from up to $6 \mathrm{~s}$ for a turning rate of $5 \% \mathrm{~s}$ at 400 $\mathrm{MHz}$, to as low as $0.04 \mathrm{~s}$ at $5 \mathrm{GHz}$ at turning rate of $60 \%$ s.

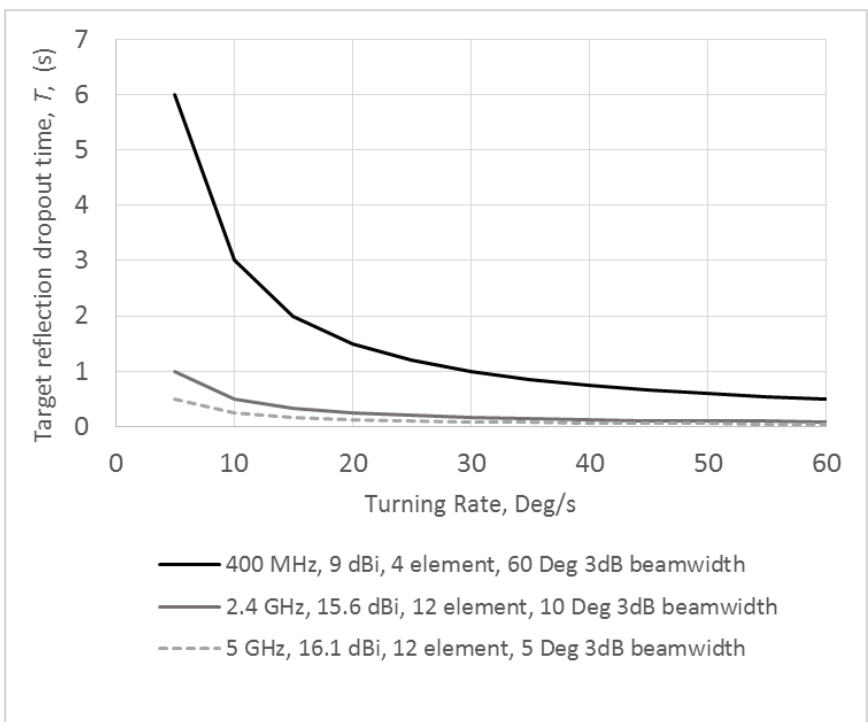

Fig. 3: Reflected signal dropout times Vs turning rate

Since hold times from PLL based retrodirective RADAR (ie no microcontroller in the tracking loop) are generally less than several milliseconds, the above clearly demonstrates the need for "track hold" circuits to provide a longer hold time. For lower frequency (eg $400 \mathrm{MHz}$ ), lower gain systems, a "track hold" circuit that simply maintains the transmit phase in the absence of RX signal is likely to be sufficient. From Fig 3, at $400 \mathrm{MHz}$, with the highest turning rate of $60 \%$, the signal reflected to the target can be maintained within the 3 $\mathrm{dB}$ points for $0.5 \mathrm{~s}$, ie the longest time that a dropout in the reflected signal can be experienced, before degradation of the reflected signal occurs.

For higher frequencies and higher gains (eg $2.4 \mathrm{GHz}, 5 \mathrm{GHz}$ ), tighter beam widths mean that the retransmitted signal to target (when using track hold) can only be maintained for very short dropout periods of the reflected signal. At the highest turning rate of $60 \%$, this equates to $80 \mathrm{~ms}$ at $2.4 \mathrm{GHz}$ and $40 \mathrm{~ms}$ at $5 \mathrm{GHz}$. To enable the retransmitted signal to stay pointed at the target for longer dropouts of the reflected signal, one potential solution is the use of a "rate of change of phase" tracking circuit. This functionality has been added to the microcontroller in Fig 1 and has been shown practically to successfully track a rate of change of phase of $20 \%$, which equates to a turning rate of $12.6 \% \mathrm{~s}$, when calculated for two antennas at $\lambda / 4$ spacing.

\section{Conclusions}

This paper has demonstrated that the addition of a "track hold" circuit to a retrodirective RADAR (a feature that was not previously available) can allow the retransmitted signal to the target to reliably continue transmission, during dropouts in the reflected signal from the target. The calculations have shown that for a lower frequency, lower gain antenna array configuration (400 MHz, 4 elements) that the track hold 
circuit can maintain the retransmitted signal, within the $3 \mathrm{~dB}$ points of the antenna array, for $0.5 \mathrm{~s}$, at a vehicle turning rate of $60 \%$ s. This shows it is likely that a track hold circuit will suffice for lower frequency, lower gain systems. At higher frequencies and higher gains (eg $2.4 \mathrm{GHz}, 5 \mathrm{GHz}$ ), the retransmitted signal to target (when using track hold) can only be maintained for $80 \mathrm{~ms}$ at $2.4 \mathrm{GHz}$ and $40 \mathrm{~ms}$ at $5 \mathrm{GHz}$ at a turning rate of $60 \%$ s. This implies that, for the higher frequency scenario, that a "rate of change of phase" tracking circuit is required.

\section{Acknowledgements}

The authors would like to thank Mr Kieran Rainey for the construction of the circuits used for this work.

\section{References}

[1] N. B. Buchanan, V. Fusco, P. Sundaralingam, "Fast Response Retrodirective Radar," IMS 2010, Anaheim, California, May 2010, Page(s): 153 - 156

[2] V. Fusco, N. Buchanan, "Angle of Arrival Detection using Retrodirective RADAR" European Microwave Conference 2010, Paris, Sep 2010

[3] N.B. Buchanan, V. Fusco, "Modulation Insensitive PLL for Tracking Antenna Applications", Microwave and Optical Technology Letters, Vol. 57, No. 6, 06.2015, p. 1286-1289

[4] "Radar Measurements of NHTSA's Surrogate Vehicle SS_V," US Department of transportation, www.nhtsa.gov

[5] E.B. Brown, E.R. Brown, "Retrodirective noisecorrelating radar at $\mathrm{X}$ band: first demonstration of small target detection," 2005 IEEE MTT-S International Microwave Symposium Digest, 12-17 June 2005

[6] V. Fusco, N. B. Buchanan, "High-Performance IQ Modulator-Based Phase Conjugator for Modular Retrodirective Antenna Array Implementation," IEEE Transactions on Microwave Theory and Techniques, Volume: 57 , Issue 10, Part 1, October 2009, Page(s): $2301-2306$

[7] V. Fusco, N. B. Buchanan "Retrodirective Array Based RADAR Front End Technology" European Conference on Antennas and Propagation 2010, 12-16 April 2010, Barcelona (Invited Paper)

[8] Hughes 9350 BGAN mobile satellite terminal specification http://www.bgansatellite.com/content/Hughes-9350Datasheet.pdf 